\title{
Dynamic behaviour of pneumatic actuators in open-loop controlled by proportional valves
}

\author{
Valentin Nicolae Cococi ${ }^{1}$, Carmen-Anca Safta $^{1^{*}}$, and Constantin Călinoiu ${ }^{1}$ \\ ${ }^{1}$ University Politehnica of Bucharest, Power Engineering Faculty, 313 Splaiul Independentei, 060042, \\ Bucharest, Romania
}

\begin{abstract}
The development of proportional and servo-mechanisms hydraulic control technique offered the premises of the development of pneumatic control, too. Despite the disadvantages of the nonlinearities appeared in the pneumatic systems (air compressibility being one of the causes), the new generation of proportional equipment made it possible to have a good quality pneumatic control system in our days. The goal of the paper is to demonstrate, by using numerical simulation technique, that the proportional valves used in pneumatic actuator drives occur at a good system dynamic at a reasonable cost as against to the directional control valve as it usually used in pneumatic actuator drives.
\end{abstract}

\section{Introduction}

The pneumatic system drives have been widely used in automatic production lines, machine tools, industrial robots, automotive industry, and a lot of other fields of activity where an automation equipment is needed. In our days $30 \%$ of automated processes and $43 \%$ of industrial robots are using pneumatic systems in closed-loop as positioning control systems, or in open-loop as pneumatic drives [1,2].

Advantages of pneumatic drives to be light weight, small-sizes, simple to install and to maintain, the robust design and operation, also to be clean and cheap, to have better working temperatures (the air must not be cooled as the hydraulic oil), make them to be preferred in many automatic applications. In the last decades the development of microelectronic technology goes to improve the static characteristics and dynamic behaviour of the pneumatic systems, despite of their nonlinearities which make them difficult to control. The gas flow itself, the gas compressibility, the aerodynamic asymmetry of gas resistance, the small viscosity coefficient and the poor lubricity of the gas are some of factors which have a strong influence in the dynamic response of a pneumatic control system [1-4].

Pneumatic systems convert the compressed air energy into mechanical energy which can be a useful mechanical work or motion. The main device of this power loop is the pneumatic actuator (piston-cylinder or rotary) [3].

Pneumatic actuators are direct-driven systems because the output rod of the linear motor (or the output motor shaft of the rotary motor) is the actuator output link [5].

\footnotetext{
* Corresponding author: $\underline{\text { safta.carmenanca@gmail.com }}$
} 
Frequently, the pneumatic actuator is controlled by an electropneumatic control valve which can be a servo valve, a proportional valve or a solenoid valve [5]. The electropneumatic valves are designed to work with one stage, two and three stages. A single stage electropneumatic control valve is a directional operated valve. The electropneumatic control valves with two and three stages are known to have one stage to pilot and amplify the pressure on the other stage. These are known as servo valves and proportional valves. The servo valves provide a higher degree of loop control and they are used in closed loop positioning system for their positioning accuracy of $\pm 0.05 \mathrm{~mm}$ [5].

The goal of the paper is to show that a pneumatic actuator controlled by a proportional valve and working in open loop can have a good dynamic behaviour to different types of electrical signal waves. Numerical simulations are made using the simulation platform offered by Simcenter Amesim with LMS Imagine.Lab Pneumatics library [6]. The numerical results are discussed, and final conclusions are underlined.

\section{Pneumatic actuator in open loop}

Starting from the classical structure of a pneumatic actuator in open loop, Figure 1, controlled by a spool type direct solenoid spring return $3 / 2$ (usually named as directional control valve, DCV), it was change the DCV with a proportional valve (Figure 2) to actuate a butterfly valve used in industrial applications.

The scheme of the pneumatic actuator controlled by the solenoid DCV will have adjustable throttles (TC1, TC2) to modify the piston velocity and two proximity sensors are attached outside of the cylinder to provide a noncontact indication of the rod piston [5, 7]. Noise dampers (or silencers, according to ISO 1219-1: 1991) are included, too. The proximity sensors and the silencers are not figured.

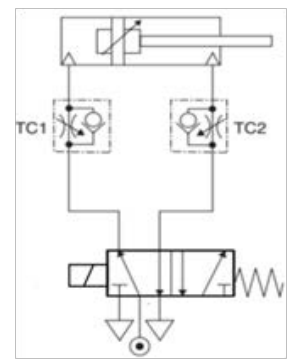

Fig. 1. Pneumatic actuator with solenoid DCV and throttle valves (TC1, TC2)

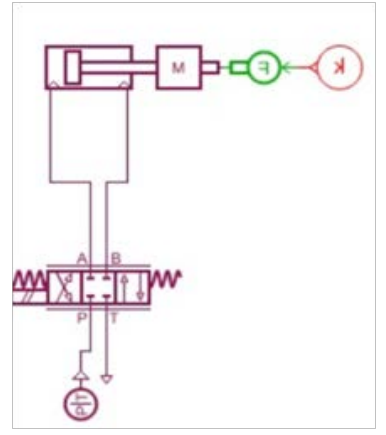

a) $4 / 3$ closed center valve

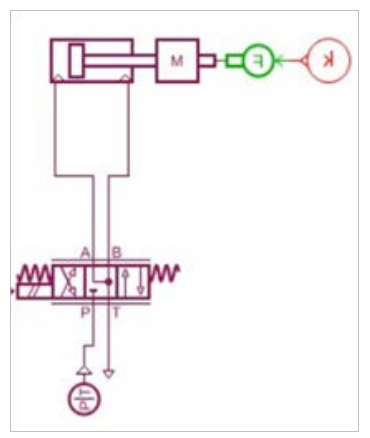

b) $4 / 3 \mathrm{ABT}$ valve

Fig. 2. Pneumatic actuator with proportional valve, Amesim simulation scheme with $M$ and $F, K$ (in the mirror) symbols for mass, and constant force. 
It is known that the open-loop pneumatic systems are sensitive to the initial conditions and not attenuate disturbances [5]. So, this kind of pneumatic systems are sensitive to parameter variations of the inputs. The parameter disturbance at the inputs will change the system outputs.

For this reason, it was proposed a pneumatic actuator controlled by a proportional valve with four ways and three positions of the spool, 4/3. The pneumatic drive is proposed in two configurations: with closed center (Figure 2, a) and with semi-opened center (Figure 2, b).

The pneumatic schemes were drawn in LMS Amesim.

\section{Pneumatic actuator parameters and signals command}

The pneumatic actuator presented in both configurations of Figure 2 has following physical characteristics of the pneumatic cylinder and proportional valve demanded by LMS Imagine.Labs Pneumatic library:

$\checkmark$ for the double-acting pneumatic cylinder, the piston diameter is $100 \mathrm{~mm}$, the rod diameter is $60 \mathrm{~mm}$, the length of the stroke is $0.3 \mathrm{~m}$, the dead volume in the cylinder is $60 \mathrm{~cm}^{3}$, the total mass being moved is $50 \mathrm{~kg}$, the viscous friction coefficient is 70 $\mathrm{Ns} / \mathrm{m}$, the temperature in the input and output ports of the pneumatic cylinder is 293.15 $\mathrm{K}$;

$\checkmark$ for the spool type 4/3 proportional valve, the area of control orifices is $7 \mathrm{~mm}^{2}$, the valve natural frequency is $80 \mathrm{~Hz}$, the valve rated current is $10 \mathrm{~mA}$, the valve damping ratio is 0.8 and the flow coefficient is 0.72 .

The pneumatic system is supplied with compressed air at a pressure of 7 barA; the pressure in the inactive cylinder chamber is 2 barA and the external temperature is the same as in the cylinder chambers. The pneumatic lines are one meter long and the inside diameter is $10 \mathrm{~mm}$.

It was considered three types of signal waves command applied to the electromagnetic device of the proportional valve, with the characteristics plotted in Figure 3.

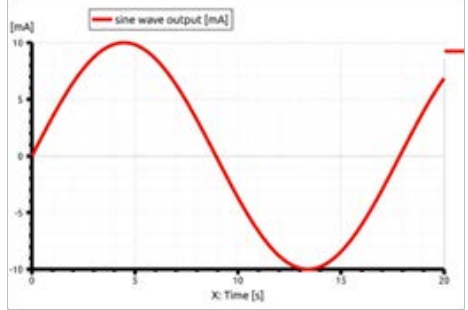

a)

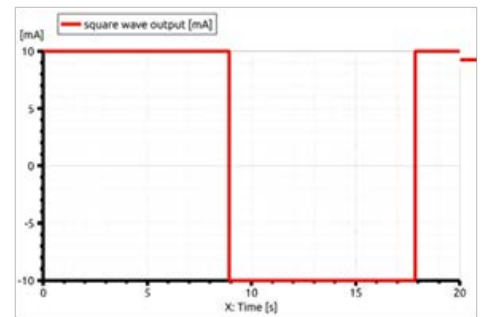

b)

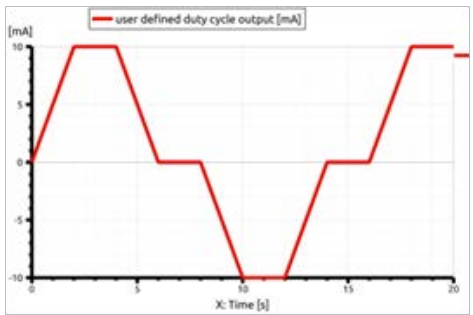

c)

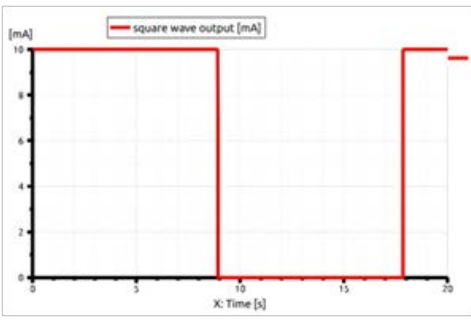

d)

Fig. 3. Three types of signal command applied to the proportional valve: a) sine wave, b) square wave; c) trapezoidal signal; d) demi-square wave. 
The sine and square wave profile signal command (Figure 3, a and b) have the amplitude $\pm 10 \mathrm{~mA}$ and the wave frequency of $0.056 \mathrm{~Hz}$. The trapezoidal profile (Figure 3, c) has the same amplitude of $\pm 10 \mathrm{~mA}$, the same period of $18 \mathrm{~s}$ but with the acceleration and deceleration time of $2 \mathrm{~s}$ each and a time of $2 \mathrm{~s}$ each for the steady state. The demi-square wave (Figure 3,d) has the amplitude $+10 \mathrm{~mA}$ and $18 \mathrm{~s}$ period. The most usual action time diagram for a solenoid DCV has a trapezoidal profile, and this is the reason to use this kind of control signal in this study.

\section{Numerical simulations of pneumatic actuator behaviour}

Numerical simulations are presented using data mentioned above. In the numerical simulation model, a constant resistant force of $3846 \mathrm{~N}$ was considered in all studied cases. The resistant force was calculated regarding the application in water butterfly valve.

Are plotted the rod displacement against time, the velocity, acceleration and pressures evolution in cylinder chambers, for all the three signals applied in each of the pneumatic actuator scheme in Figure 2. To a better follow up of the numerical results, all six working cases are named as:

$\checkmark$ PA-CC-PV-SINW, pneumatic actuator with closed center proportional valve and sine wave input, results were plotted in Figure 4;

$\checkmark$ PA-CC-PV-SQRW, pneumatic actuator with closed center proportional valve and square wave input, results were plotted in Figure 6;

$\checkmark$ PA-CC-PV-TRAP, pneumatic actuator with closed center proportional valve and trapezoidal input, results were plotted in Figure 8;

$\checkmark$ PA-ABT-PV-SINW, pneumatic actuator with ABT proportional valve and sine wave input, results were plotted in Figure 5;

$\checkmark$ PA-ABT-PV-SQRW, pneumatic actuator with ABT proportional valve and square wave input, results were plotted in Figure 7;

$\checkmark$ PA-ABT-PV-TRAP, pneumatic actuator with ABT proportional valve and trapezoidal input, results were plotted in Figure 9;

Each figure from 4 to 9 has five graphical representations denoted with (a) for the numerical simulation model, (b) for rod displacement against time, (c) for rod velocity against time, (d) for rod acceleration against time and (e) for pressure of port one and two of the big and small cylinder chambers.

From the plotted results some general observations can be done.

All displacements of the rod have three specific parts: the increase part of time, when the rod extends and gets the stroke value of $0.3 \mathrm{~m}$; the rest period of "rest" at the end of the stroke with zero velocities; the decrease part of time when the rod is retracted in the cylinder. In all six cases after the expand displacement, the time of rod restart (in the retract displacement) is long. The rod is no more moving, although the electromagnetic device of the proportional valve is receiving the electric signal command.

Numerical simulations highlight the compressibility of air flow. The time delay can be observed in the dynamic response of the pneumatic actuator having closed center valve control with sine wave and trapezoidal wave command (Fig. 4 and Fig. 8). In the case of pneumatic actuator with $\mathrm{ABT}$ valve control and the same types of command signals, the time delay is observed, too (Fig. 5 and Fig. 9).

Compared with these cases, the pneumatic actuator having square wave command will not have a delay time in the movement, not even when the pneumatic cylinder is command by proportional valve with close center, nor when it is used an ABT control valve (Figure 6 and Figure 7).

The delays are better highlighted by the velocity diagrams. 
Also, it is remarked the asymmetric evolution in the extend rod displacement which has a long time of movement than the retract of the rod in the cylinder. It is interesting that this asymmetry is not available in the case of square wave signal (Fig. 6, Fig. 7).

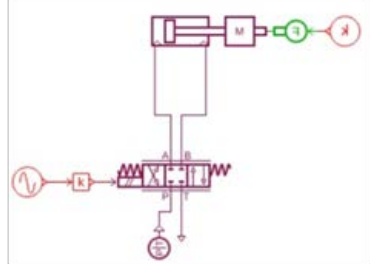

a)

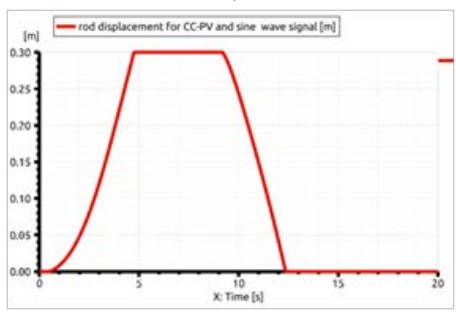

b)

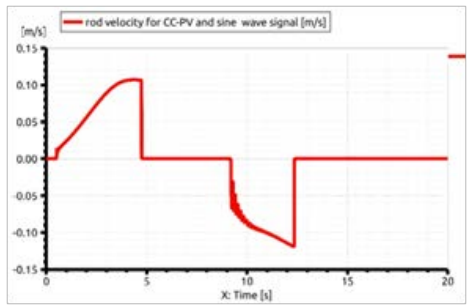

c)

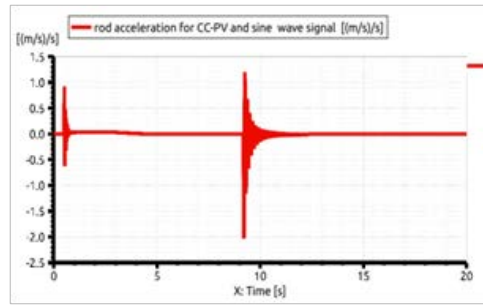

d)

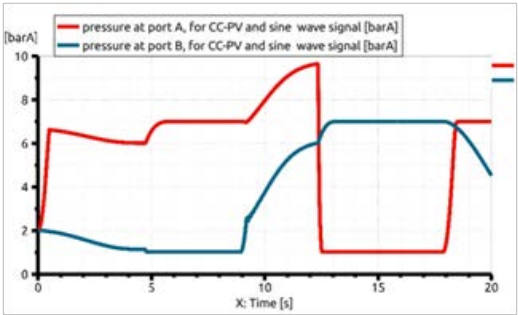

e)

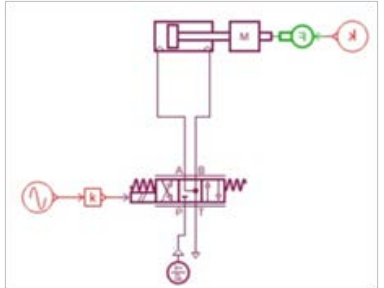

a)

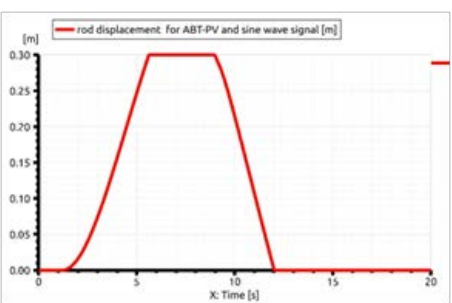

b)

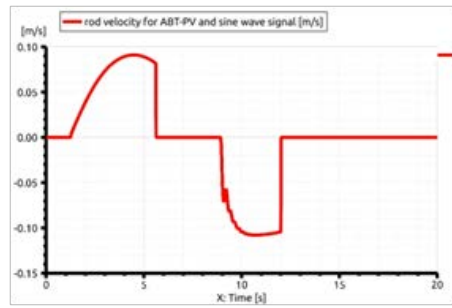

c)

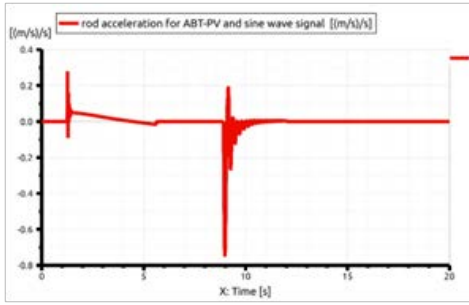

d)

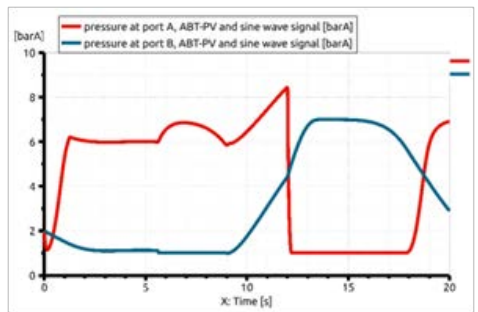

e)

4. PA-CC-PV-SINW

Fig. 5. PA-ABT-PV-SINW

In figures 4 and 5 is observed that the time delay of the pneumatic actuator with ABT control valve is longer then in case of pneumatic actuator with closed center control valve. Also is observed that the velocity evolution is smoother in the case of ABT control valve 
and the velocities are almost three times smaller than the case of closed center control valve (see Fig. 4. c and Fig. 5. c). The acceleration values are smaller in the case of ABT control valve than the close center control valve.

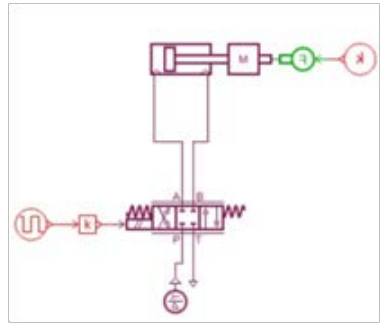

a)

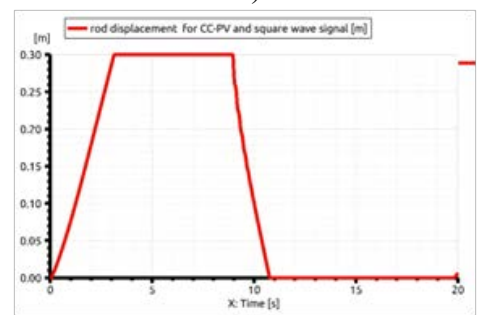

b)

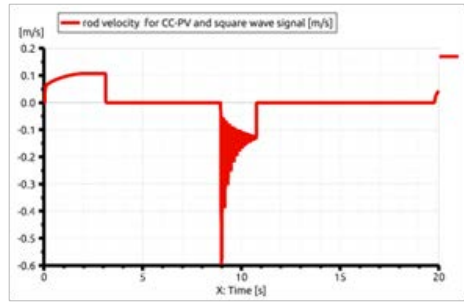

c)

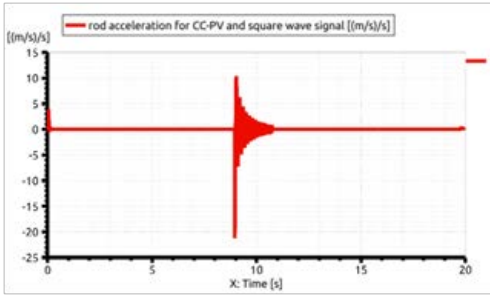

d)

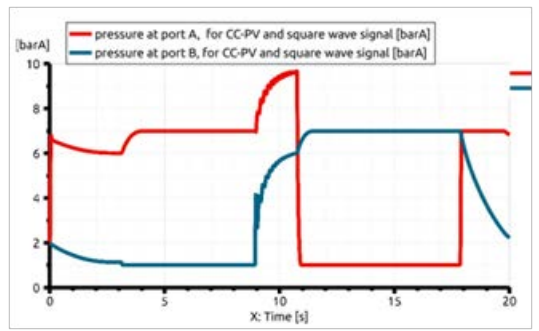

e)

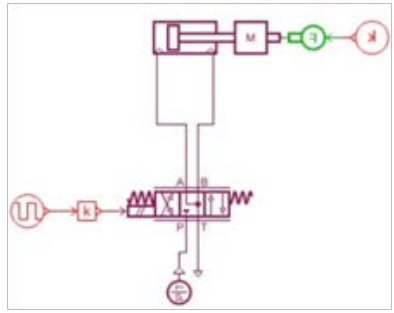

a)

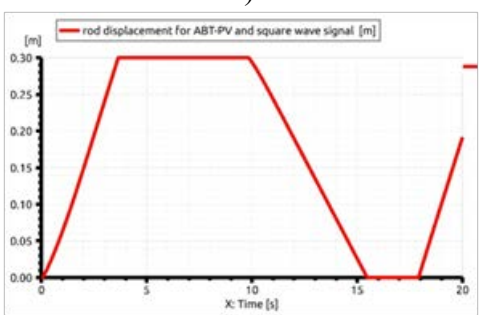

b)

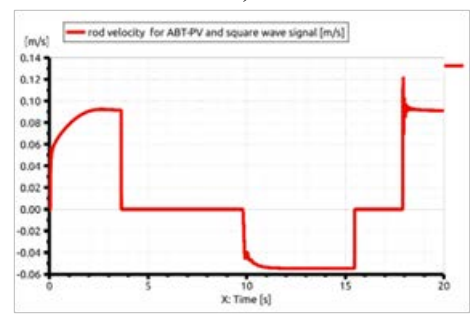

c)

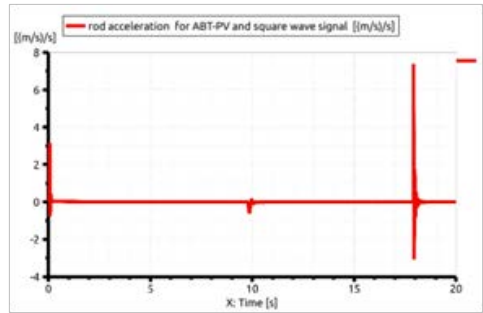

d)

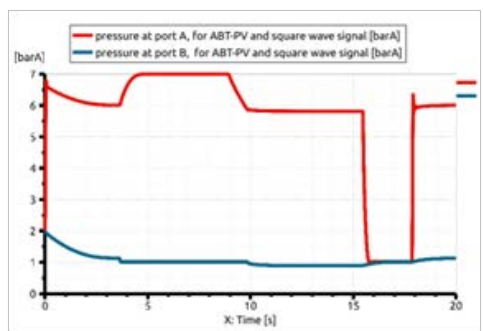

e)

Fig. 6 PA-CC-PV-SQRW

Fig. 7 PA-ABT-PV-SQRW

In figures 6 and 7 is observed the dynamic behaviour of the pneumatic actuator controlled by proportional valve at square wave signal command. In these cases, no delay 
time appear in the rod movement. It is observed that actuator tends to return under its own weight, Figure 7.e. To obtain this behaviour, the actuator controlled by ABT-PV was command by a semi-square wave signal (Figure 3,d).

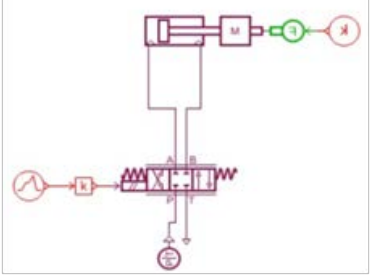

a)

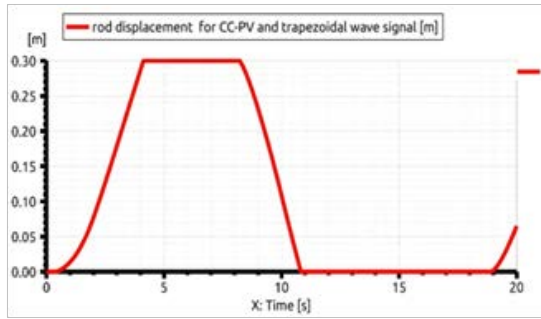

b)

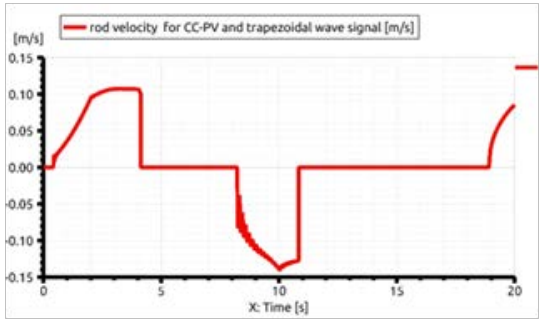

c)

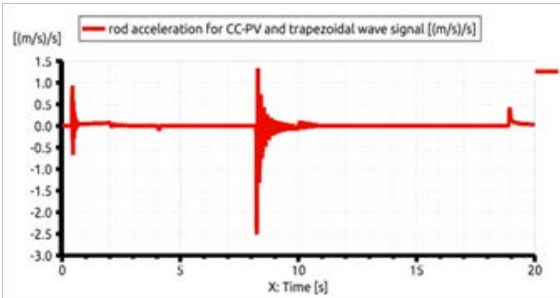

d)

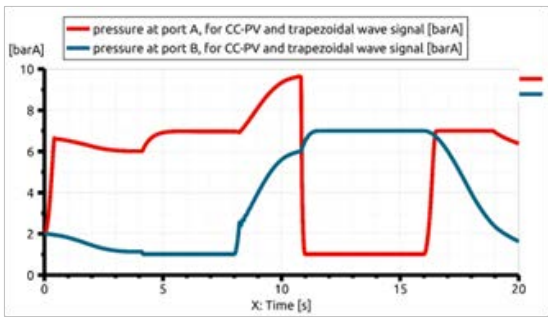

e)

Fig. 8. PA-CC-PV-TRAP

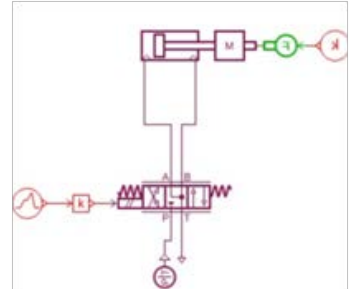

a)

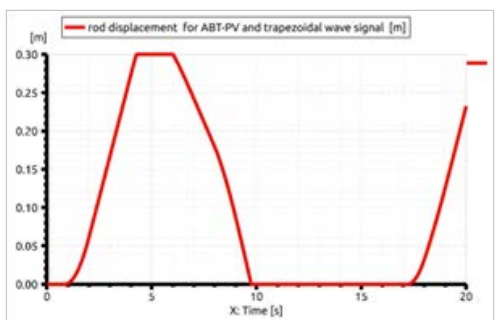

b)

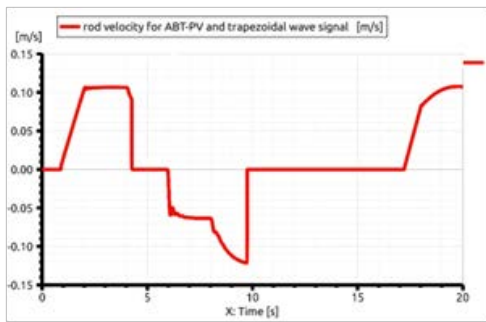

c)

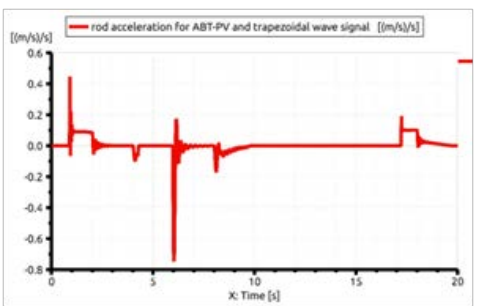

d)

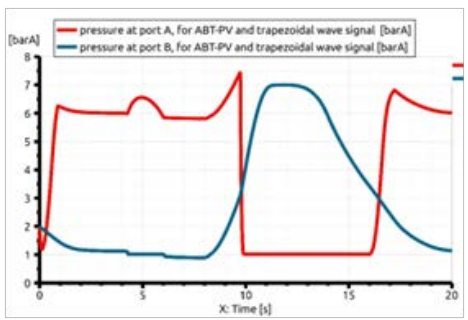

e)

Fig. 9. PA-ABT-PV-TRAP

Regarding the pressure evolution in the cylinder chambers can be observed that the same pattern is following no matter what type of pneumatic actuator is, or type of command signal. The pressure rises quickly in the bigger cylinder chamber and when the piston is at 
the end of the stroke, the pressure decreases in the meantime with the increase in pressure of the smaller chamber for a complete duty cycle (Fig. 8. e and Fig. 9. e).

\section{Conclusions}

The goal of the paper was the study of the dynamic behaviour of a pneumatic actuator in open loop, when the control valve used is a $4 / 3$ proportional valve in closed center and ABT configuration. Are used three types of signal waves for proportional valve command.

The numerical simulation results have been shown, that besides the nonlinearities known in the literature [8-11], the time delay of movement must be considered in the mathematical model of pneumatic actuator, too. Also, theoretically have been demonstrated that using proportional valve the pneumatic drive architecture is simplified. More, the dynamics of the pneumatic actuator in open loop can be controlled easily and parameters can be changed only from the electrical signal input of the proportional valve. Has been noticed in the case of the square wave signal that the values of acceleration of the piston which are almost three times bigger than other cases studied.

The study will be followed by experiments on pneumatic equipment to identify the parameters of the experiments with numerical simulation results.

Special thanks to OFRIM Group (https://ofrimgroup.com/) for all the support provide for this research.

\section{References}

1. Y. Yin, High Speed Pneumatic Theory and Technology, vol. I, Servo System, (Springer, 2019)

2. G. Chaohui, W. Chenggang, 10.5013/IJSSST.a.17.02.10 Int, (2016)

3. H. I. Ali, S. B. B. Mohd Noor, S. M. Bashi, M. H. Marhaban, A Review of Pneumatic Actuators (Modeling and Control), Australian Journal of Basic and Applied Sciences, 3(2): 440-454 (2009)

4. E. Richer, Y. Hurmuzlu, A High Performance Pneumatic Force Actuator System. Part 1 - Nonlinear Mathematical Model, ASME Journal of Dynamic Systems Measurement and Control, 122, No.3, pp. 416-425, (2001)

5. I. Krivits, G. V. Krejnin, Pneumatic Actuating Systems for automatic equipment. Structure and design, (CRC Press Press Taylor and Francis Group, 2006)

6. N. Vasiliu, D. Vasiliu, C. Călinoiu, R. Puhalschi, Simulation of fluid power systems with Simcenter Amesim, (CRC Press Taylor and Francis Group, 2018)

7. M. Galal Rabie, Fluid Power Engineering, (McGraw Hill Companies, 2009)

8. Y. Tassa, T. Wu, J. Movellan, E. Todorov, Modeling and Identification of Pneumatic Actuators, conference paper, DOI: 10.1109/ICMA.2013.6617958, https://www.researchgate.net/publication/261479950

9. G. A. Muzy, A. S. Caporali, Positioning system of a pneumatic actuator driven by proportional pressure regulator valves, Proceedings of the 4th Workshop on Innovative Engineering For Fluid Power - WIEFP 2018, ABIMAQ, São Paulo, SP, Brazil, DOI: 10.3384/ecp1815611 (2018)

10. V. Constantin, G. Belforte, O. Dontu, Mihai Avram, U.P.B. Sci. Bull., Series D, 76, Iss. 4, pp 57-68, (2014)

11. M. Sorli, L. Gastaldi, E. Codina, S. de las Heras, Dynamic analysis of pneumatic actuators, Simulation Practice and Theory 7, pp. 589-602, (1999) 